\title{
Video Performance-Debriefings and Ventilation-Refreshers Improve Quality of
}

\section{Neonatal Resuscitation.}

Christiane Skåre, ${ }^{1}$ Anne Marthe Boldingh, ${ }^{2}$ Jo Kramer-Johansen, ${ }^{1}$ Tor Einar Calisch, ${ }^{3}$ Britt Nakstad, ${ }^{2}$ Vinay Nadkarni, ${ }^{4}$ Theresa M. Olasveengen, ${ }^{5}$ Dana E. Niles. ${ }^{6}$

Institutions where the work was performed:

Oslo University Hospital and Akershus University Hospital, Norway.

Christiane Skåre, M.D.

${ }^{1}$ Norwegian National Advisory Unit for Prehospital Emergency Care (NAKOS) and Department of Anaesthesiology, Oslo University Hospital and University of Oslo, Oslo, Norway

Anne Marthe Boldingh, M.D.

${ }^{2}$ Department of Paediatric and Adolescent Medicine and Institute of Clinical Medicine, University of Oslo and Akershus University Hospital, Lørenskog, Norway

Jo Kramer-Johansen, M.D., Ph.D., Professor

${ }^{1}$ Norwegian National Advisory Unit for Prehospital Emergency Care (NAKOS) and Department of Anaesthesiology, Oslo University Hospital and University of Oslo, Oslo, Norway

Tor Einar Calisch, M.D.

${ }^{3}$ Neonatal Intensive Care Unit, Oslo University Hospital, Oslo, Norway 
Britt Nakstad, M.D., Ph.D., Professor

${ }^{2}$ Department of Paediatric and Adolescent Medicine and Institute of Clinical Medicine,

University of Oslo and Akershus University Hospital, Lørenskog, Norway

Vinay M. Nadkarni, M.D., M.S., Professor

${ }^{4}$ Department of Anesthesia, Critical Care and Pediatrics, University of Pennsylvania Perelman School of Medicine, The Children`s Hospital of Philadelphia, Philadelphia, USA

Theresa M. Olasveengen, M.D., Ph.D.

${ }^{5}$ Department of Anaesthesiology, Oslo University Hospital, Oslo, Norway

Dana E. Niles, M.S.

${ }^{6}$ Center for Simulation, Advanced Education and Innovation

The Children`s Hospital in Philadelphia, Philadelphia, USA

\section{Corresponding author:}

Christiane Skåre, Department of Anaesthesiology,

Oslo University Hospital, PB 4956 Nydalen, N-0424 Oslo, Norway

Telephone: +47 23016837, Fax: +47 23016799, Mobile: +47 92208508

Email: christiane.skare@medisin.uio.no

Word count: 2999

Abstract word count: 250

Number of figures: 2

Number of tables: 4 
Keywords: Neonatal resuscitation; Education; Debriefing; Performance-based debriefing; Insitu high-frequency simulation training; Refresher training; Positive pressure ventilation; Adherence to guidelines; Implementation; Video assessment; Clinical outcome

The study was registered at clinicaltrials.org (NCT02347241).

Institutional approval reference numbers: Oslo University Hospital: 2013/12769, Akershus University Hospital: 14032 


\begin{abstract}
Aim

Providers caring for newly born infants require skills and knowledge to initiate prompt and effective positive pressure ventilation (PPV) if the newborn does not breathe spontaneously after birth. We hypothesized implementation of high frequency/short duration deliberate practice training and post event video-based debriefings would improve process of care and decreases time to effective spontaneous respiration.
\end{abstract}

\title{
Methods
}

Pre- and post-interventional quality study performed at two Norwegian university hospitals. All newborns receiving PPV were prospectively video-recorded, and initial performance data guided the development of educational interventions. A priori primary outcome was changed from process of care using the Neonatal Resuscitation Performance Evaluation (NRPE) score to time to effective spontaneous respiration as the NRPE score could only be obtained from one site due to lack of staff resources.

\section{Results}

Over 12 months, 297 PPV-Refreshers and 52 performance debriefings were completed with 227 unique providers attending a PPV-Refresher and 93 unique providers completed a debriefing. We compared 102 PPV-events pre- to 160 PPV-events post-bundle implementation. The time to effective spontaneous respiration decreased from median $(95 \%$ confidence interval) 196 (140-237) to 144 (120-163) seconds, $\mathrm{p}=0.010$. The NRPE-score increased significantly from median $77 \%$ (75-81) pre- to $89 \%$ (86-92) post-implementation, $\mathrm{p}<0.001$. There were no significant differences in time to heart rate $>100$ beats/minute or number of newborns transferred to intensive care.

\section{Conclusion}


High frequency/short duration deliberate practice PPV psychomotor training combined with performance-focused team debriefings using video recordings of actual resuscitations may improve time to effective spontaneous breathing and adherence to guidelines during real neonatal resuscitations. 


\section{Introduction}

An estimated 3-6\% of newborns require positive pressure ventilations (PPV) immediately after birth to help the transition from intra- to extra-uterine life [1-3]. Emerging evidence suggest quality of PPV in the delivery room is uncontrolled [4], and there are concerns that excessive pressures and volumes may cause lung and brain injury [5, 6]. Poor ventilation technique reduces the effectiveness of PPV, delaying spontaneous ventilation and improvement in heart rate [7].

Providers caring for the newly born baby should be trained to initiate effective PPV. Multiple studies have demonstrated that training competency is transient, with a decline in resuscitation skills and knowledge within 3 months [8]. A Cochrane review from 2015 evaluating the effectiveness of standard neonatal resuscitation training identified low quality evidence to support our current training methods, and concluded "innovative educational methods that enhance knowledge, skills and team behaviour should be evaluated" [9]. In an effort to improve training, numerous studies suggest "just-in-time" (just prior to procedure) and "justin-place" (within the clinical environment) simulation training may be an effective concept to improve clinical skills [10-12]. Video recording has been used to evaluate teamwork and guideline compliance during both simulated and real neonatal resuscitation and provided insights to targeted education [13-17]. Video may be a feasible method to assess performance and facilitate quality improvement [18]. The role of video and debriefing to enhance knowledge, competency, behavioural skills and team work, and how to determine whether simulation training improves clinical outcomes are outlined as gaps in knowledge [19]. Datadriven performance-focused debriefings after resuscitations are recommended, but the quality of evidence is still low [18]. 
We hypothesized that the implementation of a bundled training intervention consisting of 1) high frequency/short duration deliberate practise psychomotor skill training (PPV-refresher training), and 2) post event video-based performance focused team debriefings improves process of care and decreases time to effective spontaneous respiration for newborns requiring PPV.

\section{Methods}

This prospective interventional study was approved by local institutional review boards (Oslo University Hospital (OUH) and Akershus University Hospital (AUH), Norway) as a quality improvement study contingent on prompt deletion of all videos as soon as they had been analysed. Information about the study and how to opt-out was provided to all involved health care providers and expecting mothers. Both providers and parents could opt-out by oral or written notification to the study personnel at any time during the study, at which time the videos were deleted without review. To protect healthcare provider and patient confidentiality, all videos were password protected and not accessible to anyone outside the research group and were deleted within a week after review. The study was registered at clinicaltrials.org (NCT02347241). Institutional approval reference numbers: OUH: 2013/12769, AUH: 14032.

The study was performed in the delivery units of both hospitals and was initiated with a 2.5month baseline assessment period (OUH: 15 January-02 April 2014; AUH: 28 February-14 May 2015) to record the incidence, quality and characteristics of psychomotor skill performance during neonatal resuscitation, and patient outcomes. Prior to initiation of baseline registrations staff completed a survey on self-reported competency and confidence. The results from this survey have not been published and were primarily used to consolidate 
researchers, administrators and providers perceptions of the need for an educational intervention. Non-guideline compliant practice was identified and used to develop educational interventions. Compliance issues were both technical (frequent interruptions during PPV and suboptimal bag-mask skills), and non-technical (communication, leadership and task management). The educational intervention consisted of PPV-Refreshers and educational video-based post-resuscitation performance debriefings (described below). The sequence of implementation of these two interventions were alternated between the two sites, OUH implemented post-resuscitation debriefings for the first five months, whereas AUH implemented PPV-Refreshers first. The initial implementation (OUH: 03 April-24 August 2014; AUH: 15 May-13 October 2015) was followed by a continuous post-implementation period (OUH: 25 August 2014-14 January 2015; AUH: 15 May 2015-28 February 2016) consisting of both PPV-Refreshers and monthly debriefings at both hospitals (Fig. 1). We report data from all three study phases to describe the implementation process, but only baseline and post-implementation phases are formally compared. All providers trained to initiate neonatal resuscitation were eligible for inclusion.

\section{PPV-Refreshers -High-Frequency/Short-Duration Psychomotor Skill Training}

PPV-Refreshers were performed in-situ on the resuscitation table with a newborn manikin (Newborn Lung Simulator or Baby Anne, Laerdal Medical, Stavanger, Norway) that demonstrated chest rise when appropriate volume and pressures were delivered, and a selfinflating-bag (Laerdal Medical) or T-piece resuscitator (Fisher \& Paykel Healthcare, Auckland, New Zealand).

The brief, instructor-facilitated, skill-focused sessions with PPV-Refreshers were offered on a weekly basis. A scenario chart was developed based on the training needs identified during 
the baseline period [20]. Healthcare providers caring for mothers with highest risk deliveries were identified and invited to participate in the PPV-Refresher training. Each participant was given a resuscitation scenario and instructed to demonstrate initial steps of neonatal resuscitation (dry, stimulate, assess breathing), assess need for intervention, and provide effective PPV (defined as visible chest rise and rate 30-60/minute) on the manikin for 1 minute. Additional hands-on instructions and remediation of skills were provided by the instructor, until mastery of the skills was demonstrated. The PPV-Refresher generally lasted < ten minutes.

\section{Post-Event Video-Based Performance Focused Team Debriefings}

PPV skill performance and process of care during real clinical neonatal resuscitations were recorded with video. Cameras with motion sensors (HIKVision Digital Technology Co, Ltd., Hangzhou, China) captured consecutive resuscitations, and events where PPV was delivered after delivery were included for analysis. The configurations of the video recording process, informed consent, policy and procedures have been previously described [20, 21]. Three investigators, not blinded to the study phase, transcribed and analysed all videos within a week after the recordings. The same investigator did all the NRPE-scoring. We have recently pre-validated the NRPE-score for the assessor in this study, and found a reasonable intra-rater and inter-rater reliability (median (95\% CI) Intraclass Correlation Coefficient 0.91 (0.85-0.95) and $0.76(0.53-0.88)$, respectively) [21]. Time critical interventions were reported in seconds and measured from arrival at the resuscitation table. NRPE-score, initial heart rates and respiration, ventilation parameters and time critical interventions were assessed from video. Gestational age, weight, gender, Apgar scores, and clinical outcomes were recorded from hospital records. 
Video-recorded resuscitation events with learning potential were selected, and participating team was invited to review and debrief the event. Debriefings were offered and scheduled according to the availability of multidisciplinary staff, and led by at least one study team member trained in facilitating debriefings. Sessions were structured to allow discussion of process of care, technical skills and performance. Each debriefing consisted of three parts. First, review of videos to construct an objective understanding of the scenario. Second, reflection and discussion of any perceived process of care issues, latent and overt errors, barriers to process and examples of excellence. Finally, the session was summarized, highlighting areas for improvement. The debriefing program has been previously described in detail [21].

\section{Outcome Measures}

All infants that received PPV (excluding infants that received only continuous positive airway pressure - CPAP), and were video-recorded during the different phases of the study were included. Our primary outcome measure was: 1) Time to effective spontaneous respiration (defined as; from time the infant was put on the resuscitation table to end of PPV). In infants where PPV was stopped and started again, the "final" stop in PPV was used. Infants proceeding to NICU with ongoing PPV were counted and reported separately since there was no "end of PPV" in these infants. Secondary outcome measures were: 2a) Adherence to guidelines and process of care were measured using the $\underline{\text { Neonatal }}$ Resuscitation Performance Evaluation (NRPE) score $[16,22,23]$. The criteria were modified by adding "Calling for help" and removing "free flow oxygen" from this study to account for updates to current resuscitation guidelines [24]; 2b) Time to heart rate $\geq 100 \mathrm{bpm}$ (in those with initial heart rate $<100 \mathrm{bpm}$ ); $2 \mathrm{c}$ ) Admission to NICU; 2d) Provider assigned Apgar score at 5 minutes. The NRPE-score was the a priori 
primary outcome, but due to limited staff-resources it could only be obtained from OUH and the primary outcome was changed to time to effective spontaneous respiration.

\section{Data Analysis}

Continuous data were compared with independent samples Mann-Whitney U test for two samples or Kruskal Wallis Test for more than two samples. Categorical outcome data were analysed using Pearson Chi-Square for more than two samples or Fisher's test for two samples. Statistical calculations were performed in SPSS 25.0 (SPSS Inc., IL, USA). Results are reported as numbers with percentages or medians with $95 \%$ confidence intervals $(95 \%$ CI). P-values $\leq 0.05$ were considered significant.

The total study period was set to 12 months to provide enough time for baseline recordings, interventions, and evaluation. We anticipated approximately 12500 babies to be born during this period with $500(4 \%)$ receiving PPV [2]. A sample size estimate of outcome "time to effective spontaneous respiration" (calculated upon decision to revise primary outcome) indicated that to detect a meaningful decrease in time from 180 to 120 seconds (standard deviation 120) with power of $90 \%$ and two-tailed alpha of $5 \%$, would require a sample size of 86 resuscitations pre- and post-implementation.

\section{Results}

During the one-year study period, a total of 440 of 12931 live born infants received PPV and were video-recorded and included for analysis at the two hospitals: 102 of 3285 births during the Baseline Phase (pre-implementation); 178 of 5258 during the Implementation Initiation Phase; and 160 of 4388 during the Post-Implementation Phase. Consort type diagram in Fig. 
2. Infant characteristics and resuscitation interventions were similar pre-, during and postimplementation (Table 1 and 2).

\section{Implementation of the Educational Intervention}

During the study period a total of 297 PPV-Refreshers and 52 debriefings were completed. There were 227 unique attendees (51 physicians, 169 nurses, 7 others) at the PPV-Refreshers, and $64(28 \%)$ of them participated in more than one session. At the debriefings, there were 93 unique attendees (50 physicians, 32 nurses, 11 others), and 33 (39\%) providers attended more than one debriefing. Duration of each debriefing was approximately 45 minutes. 282 of 319 eligible providers $(88 \%)$ participated in at least one of the interventions.

\section{Clinical Outcome of the Resuscitated Newborns}

Time to effective spontaneous respiration was significantly shorter post-implementation 196 (140-237) seconds compared to 144 (120-163) seconds post-implementation $(\mathrm{p}=0.010)$ (Table 3). Providers evaluated initial heart rate to be <100 bpm in 45 of 102 (44\%) newborns receiving PPV pre-implementation and in 69 of the $160(43 \%)$ newborns postimplementation. Initial heart rate was not possible to evaluate based on videos or pulse oximetry in $10(10 \%)$ pre- and $18(11 \%)$ post-implementation (Table 1$)$. In the group with heart rate $<100 \mathrm{bpm}$, time to heart rate $\geq 100 \mathrm{bpm}$ was 132 (87-161) pre- vs. 110 (97-151) seconds post-implementation $(\mathrm{p}=0.99)$. The time to evaluate first heart rate was $17(11-24)$ pre- vs. 16 (12-20) seconds post-implementation, respectively ( $\mathrm{p}=0.61)$ (Table 2). Apgar scores at 5 minutes improved significantly from 7 (7-8) pre- to $8(8-8)$ post-implementation $(\mathrm{p}=0.003)$ (Table 1). During pre-implementation period, 56 of $102(55 \%)$ newborns were transferred to NICU vs. 82 of $160(52 \%)$ newborns post-implementation $(\mathrm{p}=0.38)$. Details on morbidity and mortality can be found in table 1 . 


\section{Quality of Neonatal Resuscitation}

After implementation of PPV-refreshers and debriefings there were significantly less pauses during PPV resulting in improved ventilation fraction (defined as the fraction or proportion of time with PPV efforts) during the first 30 seconds of PPV from 85\% (73-100) pre- to 97\% (87-100) post-implementation $(\mathrm{p}=0.05)$ (Table 2$)$. The NRPE-score was only measured at OUS, where adherence to guidelines increased significantly throughout the study period from median $77 \%(75-81 \%)$ during pre-implementation to $89 \%$ (86-92\%) postimplementation $(\mathrm{p}<0.001)$. The score increased in all domains except "administration of oxygen" where the providers followed standard of care both pre- and post-implementation (Table 3).

\section{Discussion}

Implementation of a bundled training intervention consisting of PPV-Refreshers and performance-based team educational debriefings improved the quality of care and several surrogate measures of clinical outcome during real neonatal resuscitation. Time to effective spontaneous respiration improved from 196 to 144 seconds, guideline compliance score improved from $77 \%$ to $89 \%$, and there were significant improvements in 5-minute Apgar scores. Our study suggests that high-frequency/short duration deliberate practice psychomotor training combined with performance-based team educational debriefings can improve quality of care and patient outcomes.

An impact of high-frequency/short-duration deliberate practice and performance-based debriefings have individually been previously reported $[23,25,26]$, but it has been difficult to translate the effect from simulation to patient-related outcomes [27]. There are several randomized trials reporting improved performance in simulation and/or theoretical knowledge 
after simulation-based education, but none of them report improvements in clinical outcomes [28-31]. A meta-analysis from 2011 found that when compared to no intervention, technology-enhanced simulation was associated with large effects for improved knowledge, skills, and behaviour outcomes, with lesser effects for patient-related outcomes. They also found that distributing learning activities over more than one day was associated with higher effect [32].

Low-dose/high-frequency deliberate practice has been shown to increase retention of resuscitation skills otherwise known to decline within few months [25]. A mobile PPVRefresher cart, equipped with standard resuscitation equipment and an infant manikin, can be an impromptu supplement to debriefings and standard education [33]. Our instructor-led PPVRefreshers took less than ten minutes to conduct and included a short summary after the miniscenarios to reinforce vital concepts and best practice. This method of just-in-time/just-inplace training may also assist in providing low-scale simulation for maintenance of skills and may work well in healthcare systems that do not have access to simulation education centres. The surveillance cameras were also of limited cost (approx. 125 Euros each), and might be a cost-efficient alternative to trained observers. The granularity and rigour of video analysis could be adjusted to meet the specific institutional needs, and provide a better balance of burden vs. benefit for everyday clinical practice. An uncontrolled before and after-study from Tanzania reported a decrease in newborn mortality when 40-minute monthly re-trainings were combined with high frequency simulation training [34].

A meta-analysis from 2013 including 46 studies, found debriefings improve performance by approximately 20-25\% compared to control [35]. 2015 European neonatal resuscitation guidelines recommend debriefing after neonatal resuscitation [7], however, a multicentre 
audit from 2015 found that debriefing occurred in $<20 \%$ of the resuscitations [36]. Although video-debriefings are not found to be superior in simulation settings, [37, 38] use of videos were invaluable to ensure adequate reflection and self-assessment following our debriefings.

Due to implementation of the program across several departments, for various professional groups, and with constant turnover of providers, it was challenging to monitor the exact percent of providers participating in the program during the different study phases. A priori, we defined successful implementation as $>80 \%$ of staff receiving at least one PPV-Refresher. Yet, improvements in patient care were observed long before the target of $80 \%$ was reached, and there might be significant benefit from increased awareness among providers when more frequent and informal discussions are available [39]. We observed dedicated providers initiating their own PPV-Refreshers as the scenarios and equipment were left easily accessible in the resuscitation bays, as such making materials and equipment available on site may further lower the resources needed to achieve meaningful training and improvement in quality of care. However, there were also indications of non-compliant behaviour in all study-phases. Compared to other studies, the frequency of chest compressions was higher than expected after intervention ( $0.3 \%$ of all live births) [40], despite initial heart rates, Apgar scores and $\mathrm{pH}$ values being within expected ranges. This may indicate providers were overly eager to initiate compressions rather than the cohort consisting of very sick infants. It is important to consider the possibility that training interventions may have both beneficial and harmful effects, specifically; drilling resuscitation algorithms could alter the threshold for attempting advanced manoeuvres that might not always benefit patients. Careful assessment for both beneficial and potentially harmful effects should be carefully assessed during educational interventions. 
There are limitations to the study. First, this was a non-randomized interventional quality improvement study with the primary goal of improving quality of care, not a randomized controlled trial. Second, there is potential bias as the video reviewers were not blinded to the study phase. Attempts were made to mitigate this risk by having another investigator blinded to the study phase re-score a random sample of the videos. Furthermore, without widely accepted process measures to assess quality of neonatal resuscitation, various outcomes and definitions are used in the literature, and comparisons between studies are difficult. We chose to measure time to onset of spontaneous respiration, NRPE-score, time to heart rate $\geq 100$ bpm, Apgar scores, and admission to NICU as main measurement outcomes; as these are not all commonly reported, benchmarking is difficult. We did not have access to any respiration function monitors to accurately assess ventilation volumes or pressures during the resuscitations, therefore, decreased time to effective spontaneous respiration was used as an indicator of effective PPV. The measured outcomes might reflect true improved patient physiology, but it might also reflect differences in assessment of breathing and heart rate between the study phases. The study outcomes were not explicit explained to the providers, still providers might have been influenced by a desire to meet study targets.

Electrocardiogram was not routinely used during the study period, thus we had to rely on less accurate methods for heart rate monitoring such as auscultation of the heart rate and pulse oximetry. Due to limited research staff resources, we were also only able to use the validated neonatal resuscitation scoring tool in one of the two participating hospitals, and primary outcome therefore had to be changed from "the NRPE-score" to "time to effective spontaneous respiration”. Finally, process and outcomes measures were general, and restricted to information that could be obtained from medical records, standard monitors and video recordings. 


\section{Conclusion}

A bundled educational program consisting of high-frequency/short-duration, deliberate practice PPV skill refresher training and video performance-focused educational debriefing can decrease time to effective spontaneous respiration and increase adherence to guidelines (NRPE-score) during neonatal resuscitation. The results from this study may inform the design of future evaluations of resuscitation training interventions.

Conflict of interest statement: C. Skåre: None. T.E Calisch: None. B. Nakstad: None. J. Kramer-Johansen: None. A.M Boldingh, D. Niles and T.M Olasveengen have received research support from the Laerdal Foundation for Acute Medicine, Stavanger, Norway.

Acknowledgments: The study was supported by grants from South Eastern Health Authority. Dana Niles, Anne Marthe Boldingh and Theresa M. Olasveengen received grant support from Laerdal Foundation for Acute Medicine. The study sponsors had no involvement in the study design, in the collection, analysis, interpretation of data or in writing of the manuscript. We thank every health personnel and delivering mother who made it possible to obtain valuable data. 


\section{References}

[1] Lee AC, Cousens S, Wall SN, Niermeyer S, Darmstadt GL, Carlo WA, et al. Neonatal resuscitation and immediate newborn assessment and stimulation for the prevention of neonatal deaths: a systematic review, meta-analysis and Delphi estimation of mortality effect. BMC Public Health. 2011;11 Suppl 3:S12.

[2] Skare C, Kramer-Johansen J, Steen T, Odegaard S, Niles DE, Nakstad B, et al. Incidence of Newborn Stabilization and Resuscitation Measures and Guideline Compliance during the First Minutes of Life in Norway. Neonatology. 2015;108:100-7.

[3] Niles DE, Cines C, Insley E, Foglia EE, Elci OU, Skare C, et al. Incidence and characteristics of positive pressure ventilation delivered to newborns in a US tertiary academic hospital. Resuscitation. 2017;115:102-9.

[4] Barton SK, Tolcos M, Miller SL, Christoph-Roehr C, Schmolzer GM, Moss TJ, et al. Ventilation-Induced Brain Injury in Preterm Neonates: A Review of Potential Therapies. Neonatology. 2016;110:155-62.

[5] Polglase GR, Miller SL, Barton SK, Baburamani AA, Wong FY, Aridas JD, et al. Initiation of resuscitation with high tidal volumes causes cerebral hemodynamic disturbance, brain inflammation and injury in preterm lambs. PLoS One. 2012;7:e39535.

[6] Schmolzer GM, Te Pas AB, Davis PG, Morley CJ. Reducing lung injury during neonatal resuscitation of preterm infants. J Pediatr. 2008;153:741-5.

[7] Wyllie J, Bruinenberg J, Roehr CC, Rudiger M, Trevisanuto D, Urlesberger B. European Resuscitation Council Guidelines for Resuscitation 2015: Section 7. Resuscitation and support of transition of babies at birth. Resuscitation. 2015;95:249-63.

[8] Patel J, Posencheg M, Ades A. Proficiency and retention of neonatal resuscitation skills by pediatric residents. Pediatrics. 2012;130:515-21.

[9] Dempsey E, Pammi M, Ryan AC, Barrington KJ. Standardised formal resuscitation training programmes for reducing mortality and morbidity in newborn infants. Cochrane Database Syst Rev. 2015:Cd009106.

[10] Braga MS TM, Rhoads JM, Cacchio MP, Auerbach M, Nishisaki A, Larson RJ. Effect of just-in-time simulation training on provider performance and patient outcomes for clinical procedures: a systematic review. BMJ STEL. October 5, 2015 ed2015. p. 1-9.

[11] Cheng A, Overly F, Kessler D, Nadkarni VM, Lin Y, Doan Q, et al. Perception of CPR quality: Influence of CPR feedback, Just-in-Time CPR training and provider role.

Resuscitation. 2015;87:44-50.

[12] Scholtz AK, Monachino AM, Nishisaki A, Nadkarni VM, Lengetti E. Central venous catheter dress rehearsals: translating simulation training to patient care and outcomes. Simul Healthc. 2013;8:341-9.

[13] Thomas EJ, Sexton JB, Lasky RE, Helmreich RL, Crandell DS, Tyson J. Teamwork and quality during neonatal care in the delivery room. J Perinatol. 2006;26:163-9.

[14] Schilleman K, Siew ML, Lopriore E, Morley CJ, Walther FJ, Te Pas AB. Auditing resuscitation of preterm infants at birth by recording video and physiological parameters. Resuscitation. 2012;83:1135-9.

[15] Carbine DN, Finer NN, Knodel E, Rich W. Video recording as a means of evaluating neonatal resuscitation performance. Pediatrics. 2000;106:654-8.

[16] Gelbart B, Hiscock R, Barfield C. Assessment of neonatal resuscitation performance using video recording in a perinatal centre. J Paediatr Child Health. 2010;46:378-83.

[17] Shivananda S, Twiss J, El-Gouhary E, El-Helou S, Williams C, Murthy P, et al. Video recording of neonatal resuscitation: A feasibility study to inform widespread adoption. World J Clin Pediatr. 2017;6:69-80. 
[18] Finn JC, Bhanji F, Lockey A, Monsieurs K, Frengley R, Iwami T, et al. Part 8: Education, implementation, and teams: 2015 International Consensus on Cardiopulmonary Resuscitation and Emergency Cardiovascular Care Science with Treatment Recommendations. Resuscitation. 2015;95:e203-24.

[19] Perlman J, Kattwinkel J, Wyllie J, Guinsburg R, Velaphi S, Nalini Singhal for the Neonatal ITFG. Neonatal resuscitation: in pursuit of evidence gaps in knowledge. Resuscitation. 2012;83:545-50.

[20] Skare C, Boldingh AM, Nakstad B, Calisch TE, Niles DE, Nadkarni VM, et al. Ventilation fraction during the first 30s of neonatal resuscitation. Resuscitation. 2016;107:2530 .

[21] Skare C, Calisch TE, Saeter E, Rajka T, Boldingh AM, Nakstad B, et al. Implementation and effectiveness of a video-based debriefing programme for neonatal resuscitation. Acta Anaesthesiol Scand. 2018.

[22] van der Heide PA, van Toledo-Eppinga L, van der Heide M, van der Lee JH. Assessment of neonatal resuscitation skills: a reliable and valid scoring system. Resuscitation. 2006;71:212-21.

[23] Sawyer T, Sierocka-Castaneda A, Chan D, Berg B, Lustik M, Thompson M. Deliberate practice using simulation improves neonatal resuscitation performance. Simul Healthc. 2011;6:327-36.

[24] NRR. Resuscitering av nyfødte. Norsk Resuscitasjonstråd. 2014. (Accessed 1 January 2014, at http://nrr.org/no/)

[25] Niles D, Sutton RM, Donoghue A, Kalsi MS, Roberts K, Boyle L, et al. "Rolling Refreshers": A novel approach to maintain CPR psychomotor skill competence.

Resuscitation. 2009;80:909-12.

[26] Nadler I, Sanderson PM, Van Dyken CR, Davis PG, Liley HG. Presenting video recordings of newborn resuscitations in debriefings for teamwork training. BMJ Qual Saf. 2011;20:163-9.

[27] Cheng A, Lockey A, Bhanji F, Lin Y, Hunt EA, Lang E. The use of high-fidelity manikins for advanced life support training--A systematic review and meta-analysis.

Resuscitation. 2015;93:142-9.

[28] Rubio-Gurung S, Putet G, Touzet S, Gauthier-Moulinier H, Jordan I, Beissel A, et al. In Situ Simulation Training for Neonatal Resuscitation: An RCT. Pediatrics. 2014.

[29] Lee MO, Brown LL, Bender J, Machan JT, Overly FL. A medical simulation-based educational intervention for emergency medicine residents in neonatal resuscitation. Acad Emerg Med. 2012;19:577-85.

[30] Bruno CJ, Angert R, Rosen O, Lee C, Vega M, Kim M, et al. Simulation as a tool for improving acquisition of neonatal resuscitation skills for obstetric residents. J Matern Fetal Neonatal Med. 2016;29:2625-9.

[31] Bender J, Kennally K, Shields R, Overly F. Does simulation booster impact retention of resuscitation procedural skills and teamwork? J Perinatol. 2014;34:664-8.

[32] Cook DA, Hatala R, Brydges R, Zendejas B, Szostek JH, Wang AT, et al. Technologyenhanced simulation for health professions education: a systematic review and meta-analysis. JAMA 2011;306:978-88.

[33] Sutton RM, Niles D, Meaney PA, Aplenc R, French B, Abella BS, et al. Low-dose, highfrequency CPR training improves skill retention of in-hospital pediatric providers. Pediatrics. 2011;128:e145-51.

[34] Mduma E, Ersdal H, Svensen E, Kidanto H, Auestad B, Perlman J. Frequent brief on-site simulation training and reduction in 24-h neonatal mortality-An educational intervention study. Resuscitation. 2015;93:1-7. 
[35] Tannenbaum SI, Cerasoli CP. Do team and individual debriefs enhance performance? A meta-analysis. Hum Factors. 2013;55:231-45.

[36] Edwards EM, Soll RF, Ferrelli K, Morrow KA, Suresh G, Celenza J, et al. Identifying improvements for delivery room resuscitation management: results from a multicenter safety audit. Matern Health Neonatol Perinatol. 2015;1:2.

[37] Savoldelli GL, Naik VN, Park J, Joo HS, Chow R, Hamstra SJ. Value of debriefing during simulated crisis management: oral versus video-assisted oral feedback.

Anesthesiology. 2006;105:279-85.

[38] Byrne AJ, Sellen AJ, Jones JG, Aitkenhead AR, Hussain S, Gilder F, et al. Effect of videotape feedback on anaesthetists' performance while managing simulated anaesthetic crises: a multicentre study. Anaesthesia. 2002;57:176-9.

[39] Paradis E, Sutkin G. Beyond a good story: from Hawthorne Effect to reactivity in health professions education research. Med Educ. 2017;51:31-9.

[40] Perlman JM, Risser R. Cardiopulmonary resuscitation in the delivery room. Associated clinical events. Arch Pediatr Adolesc Med. 1995;149:20-5. 
Fig. 1.

Overview of the study phases

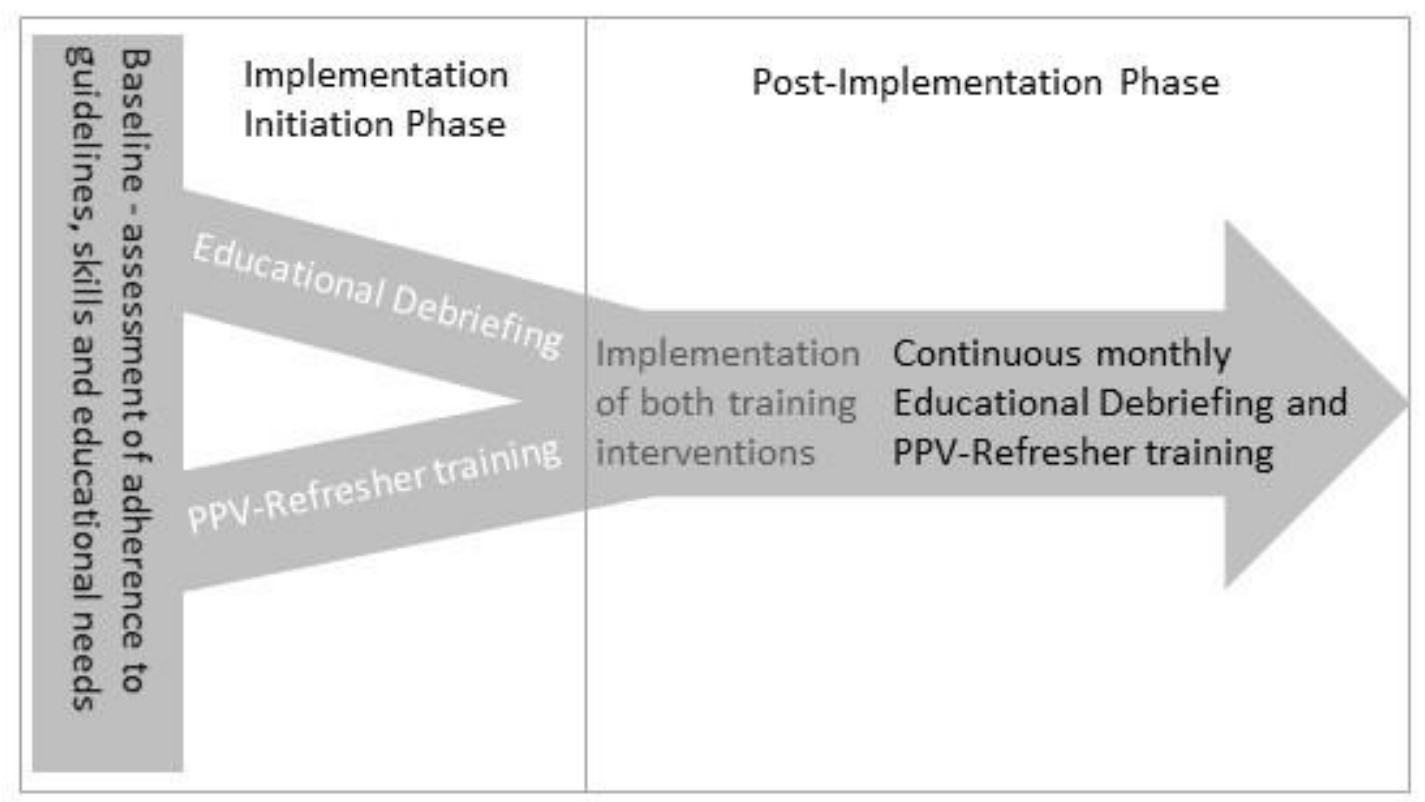


Fig. 2.

Description of the study cohort

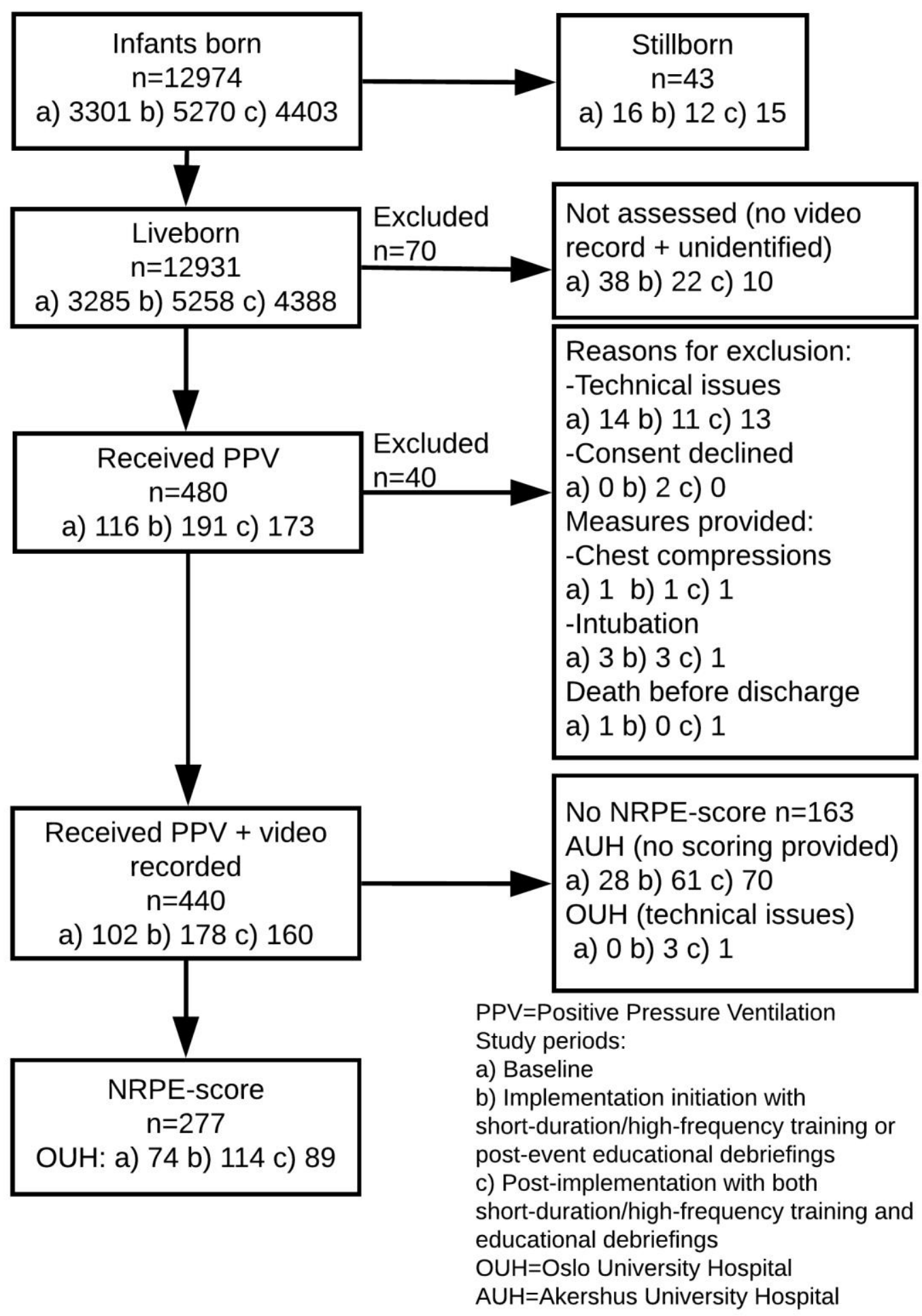


Table 1 Demographics and outcome in infants that received positive pressure ventilation (PPV) during the different phases of the study.

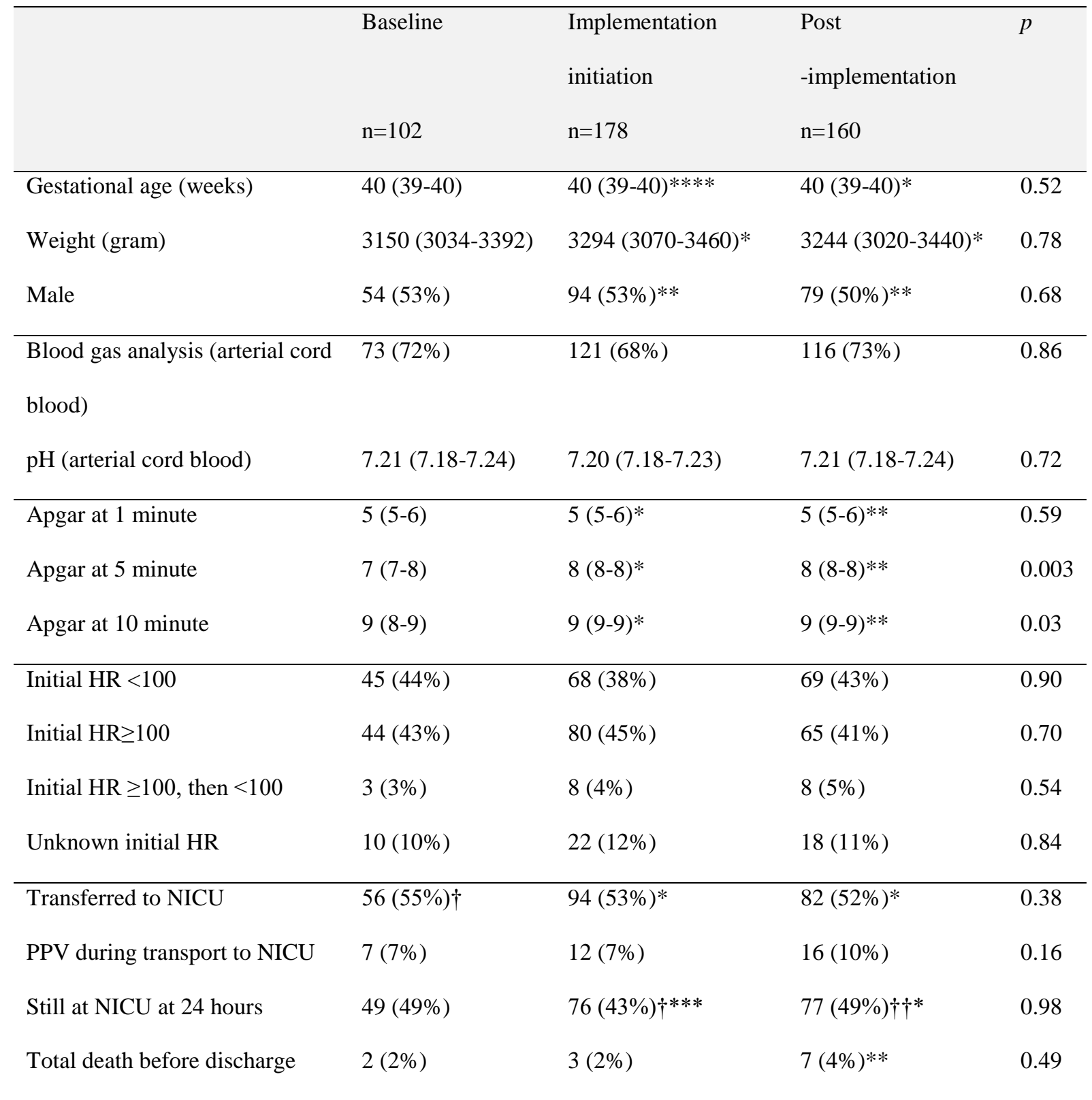

Data are given as median (95\% confidence interval) or number (\%). "Implementation initiation" reflects the period with a single intervention with either PPV-Refresher or educational debriefings. The "Postimplementation" reflects the period with continuous interventions with both PPV-Refreshers and educational debriefings at both hospitals. Comparisons of continuous data between baseline and post-implementation were done with independent samples Mann-Whitney-U test and categorical data were analysed using Pearson ChiSquare or Fisher`s Exact Test. NICU=Newborn Intensive Care Unit. *One infant missing. **Two infants missing. ***Three infants missing. ****Four infants missing. $\dagger$ One death. $\dagger \dagger$ Two deaths. 
Table 2 Advanced resuscitation measures in live born infants during the different phases of the study.

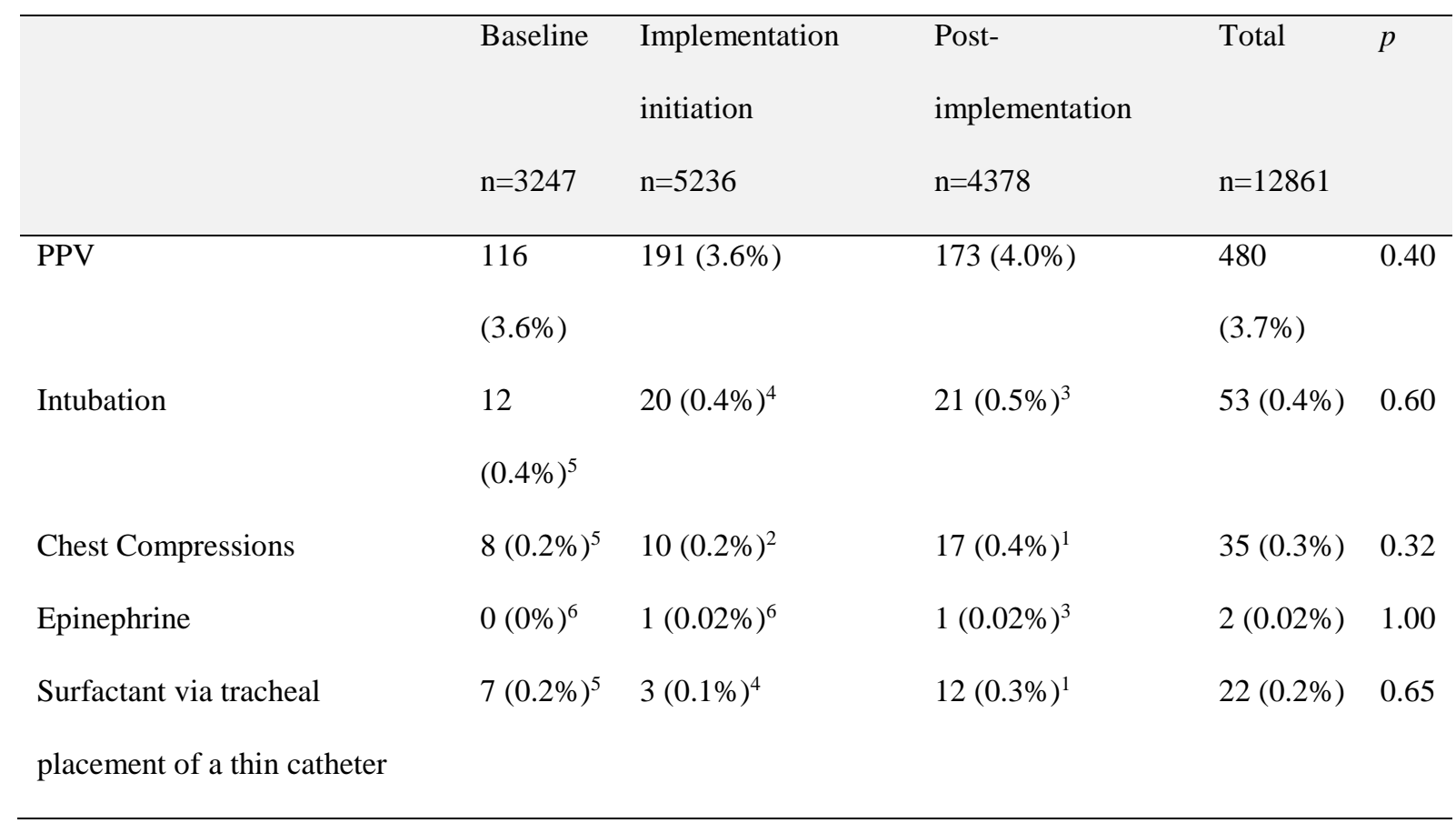

Data are given as number (\%). "Implementation initiation" reflects the period with a single intervention with either PPV-Refresher or educational debriefings. The "Post-implementation" reflects the period with continuous interventions with both PPV-Refreshers and educational debriefings at both hospitals. Comparisons of categorical data between baseline and post-implementation were analysed using Fisher`s Exact Test. There were limited information about the advanced resuscitation measures in some of the infants: ${ }^{1}=1$ infant, ${ }^{2}=2$ infants, $3=3$ infants etc. 
Table 3. The effect of implementation of high-frequency/short-duration deliberate practice skill training and post-event live capture video-based performance focused team debriefing on ventilation measures and heart rate (HR).



\begin{tabular}{|c|c|c|c|c|}
\hline \multicolumn{5}{|c|}{ Total duration of (seconds): } \\
\hline -PPV without pauses^ & $64(45-99)$ & $59(43-68)$ & $60(48-73)$ & 0.34 \\
\hline -Pauses in $\mathrm{PPV}^{\wedge}$ & $30(17-51)$ & $22(12-27)$ & $16(8-22)$ & 0.02 \\
\hline
\end{tabular}

Data are given as median (95\% confidence interval) or number (\%). "Implementation initiation" reflects the period with a single intervention with either PPV-Refresher or educational debriefings. The "Post-

implementation" reflects the period with continuous interventions with both PPV-Refreshers and educational debriefings at both hospitals. Comparisons of categorical data between baseline and post-implementation were done with independent samples Mann-Whitney U test and categorical data were analysed using Pearson ChiSquare. PPV=Positive Pressure Ventilation. ${ }^{\wedge}$ Excluded 34 infants transported to neonatal intensive care (NICU) with ongoing PPV +1 death. *One infant missing. **Two infants missing. Ventilation fraction is the cumulative fraction of time with PPV efforts excluding pauses in infants without spontaneous breathing. No infants were intubated within the first minute of arrival to the resuscitation table so time taken to intubate was not included as an interruption in initial ventilation. 
Table 4 The effect of implementation of high-frequency/short-duration deliberate practice skill training and post-event live capture video-based performance focused team debriefing on guidelines compliance measured by modified NRPE-score.

\begin{tabular}{lllll}
\hline NRPE-score & Baseline & Implementation & Post-implementation & $p$ \\
(\% of max. possible score) & & initiation & \\
& $\mathrm{n}=74$ & $\mathrm{n}=114$ & $\mathrm{n}=89$ & $<0.001$ \\
\hline Group function/communication & $88(75-90)$ & $92(83-100)$ & $100(100-100)$ & $<0.001$ \\
Preparation and initial steps & $75(70-80)$ & $88(80-90)$ & $90(88-100)$ & $<0.001$ \\
Communication of heart rate & $50(50-75)$ & $75(50-75)$ & $100(75-100)$ & 0.15 \\
Administration of oxygen & $100(100-100)$ & $100(100-100)$ & $90(90-90)$ & $<0.001$ \\
Positive pressure ventilation & $70(67-75)$ & $82(80-90)$ & $89(86-92)$ & $<0.001$ \\
\hline Total & $77(75-81)$ & $84(81-87)$ & & \\
\hline
\end{tabular}

Scores are reported as a percent of the maximum possible score in each case (sum score/max. score). The scores are given in median percent with $95 \%$ confidence interval. NRPE-score were only assessed at one of the participating hospitals (OUH). At this hospital, the implementation was initiated by debriefing, but both intervention were provided during the post-implementation phase. Comparisons of continuous data between baseline and post-implementation with PPV-Refreshers and debriefing were done with independent samples Mann-Whitney-U test. 